Nuclear Physics A438 (1985) 15-28

(C) North-Holland Publishing Company

\title{
MULTILEVEL DESCRIPTION OF THE Rh ISOTOPES IN THE INTERACTING BOSON-FERMION MODEL
}

\author{
J. JOLIE, P. VAN ISACKER, K. HEYDE, J. MOREAU, \\ G. VAN LANDEGHEM and M. WAROQUIER \\ Laboratorium voor Kernfysica, Proeftuinstraat 86, B-9000 Gent, Belgium \\ and \\ O. SCHOLTEN
}

National Superconducting Cyclotron Laboratory and Department of Physics and Astronomy, Michigan State University, East Lansing, MI 48824, USA

Received 26 October 1984

(Revised 5 December 1984)

\begin{abstract}
We present a multilevel calculation within the framework of the interacting boson-fermion model (IBFM-1) of the odd-mass ${ }^{101-109} \mathrm{Rh}$ isotopes. We calculate both positive- and negativeparity states of the same hamiltonian, starting from the $\lg _{9 / 2}, 2 \mathrm{p}_{1 / 2}, 2 \mathrm{p}_{3 / 2}, 1 \mathrm{f}_{5 / 2}$ and $2 \mathrm{~d}_{5 / 2}$ singleparticle orbitals. We discuss energy spectra, electromagnetic E2 transition rates and one-nucleon transfer properties. We also discuss the influence of the $1 f_{7 / 2}$ orbital on the one-nucleon transfer properties. The results of the calculation for the negative-parity states are compared with the results of the $U(6 / 12)$ supersymmetry scheme of the interacting boson model.
\end{abstract}

\section{Introduction}

In the most naive shell-model description of the odd-mass $\mathrm{Rh}$ nuclei, a $\left(\mathrm{g}_{\frac{2}{2}}\right)^{-5}$ five-proton hole cluster outside the $Z=50 \mathrm{Sn}$ core has to be considered. These configurations mainly determine the low-lying positive-parity levels. Besides these positive-parity levels many negative-parity levels are observed, arising from the $2 \mathrm{p}_{\frac{1}{2}}, 2 \mathrm{p}_{\frac{1}{2}}$ and $1 \mathrm{f}_{\frac{3}{2}}$ proton orbitals. Shell-model calculations in a large configuration space, taking into account both the $2 \mathrm{p}_{\frac{1}{2}}, 2 \mathrm{p}_{\frac{1}{2}}, 1 \mathrm{f}_{\frac{3}{2}}$ and $1 \mathrm{~g}_{\frac{\mathrm{g}}{2}}$ proton single-particle states, are numerically unfeasible. Moreover, the neutron-rich $\mathrm{Rh}$ nuclei around $N \simeq 60$ are situated in a region where evidenc. exists for large quadrupoledeformed shapes ').

In the light of these considerations one should start from a description where the interplay between collective and single-particle degrees of freedom is considered when studying the $\mathrm{Rh}$ isotopes. In recent years it has been shown that the interacting boson model ${ }^{2-4}$ ) is highly succesful in describing low-lying collective 
quadrupole excitations in heavy and medium-heavy nuclei. Calculations for eveneven nuclei, using the two versions of the model, the IBM-1 [refs. ${ }^{2-4}$ )] and IBM-2 $\left[\right.$ refs. $\left.\left.{ }^{5,6}\right)\right]$ (in which one distinguishes between neutron and proton bosons), have shown that it can be used to describe chains of isotopes or isotones in several regions of the mass table.

In the interacting boson-fermion model (IBFM-1) [ refs. $\left.\left.^{7,8}\right)\right]$, an extention to odd-mass nuclei, one couples the odd particle with the underlying even-even core, depicted as an interacting system of s- and d-bosons. In this model there is no distinction made between neutrons and protons. This IBFM-1 has only been applied to a few series of isotopes and the odd particle was mostly restricted to occupy only one single-particle orbital. Furthermore, when calculating positiveand negative-parity levels the calculations are carried out separately, even in multilevel calculations ${ }^{9}$ ).

In the present article, we study both positive- and negative-parity levels in $101-109 \mathrm{Rh}$ with the same hamiltonian. It was found possible to describe with a smooth-changing boson-fermion interaction, mostly depending on the even-even core nuclei, the $\mathrm{Rh}$ isotopes from neutron-deficient to neutron-rich.

\section{The interacting boson-fermion model (IBFM-1)}

\subsection{THE IBFM-1 HAMILTONIAN}

In the IBFM-1, the odd-mass nucleus is described by the coupling of an odd particle (or hole) with the underlying even-even core, the latter described by the IBM-1. In the present calculation the odd particle can occupy the $1 g_{\frac{2}{2}}, 2 p_{\frac{1}{2}}, 2 p_{\frac{1}{2}}, 1 f_{\frac{3}{2}}$ and $2 \mathrm{~d}_{\frac{5}{2}}$ orbitals. The hamiltonian describing the odd-even nucleus can be depicted as follows:

$$
H=H_{\mathrm{B}}+H_{\mathrm{F}}+V_{\mathrm{BF}}
$$

where $H_{\mathrm{B}}$ is the IBM-1 hamiltonian, used for the even-even core nucleus. The fermion degrees of freedom are given by

$$
H_{\mathbf{F}}=\sum_{j} E_{j} \hat{n}_{j}
$$

The summation runs over all possible single-particle orbitals to be considered in the odd-mass model space. $E_{j}$ denotes the quasiparticle energy of these orbitals. The boson-fermion interaction is denoted by $V_{\mathrm{BF}}$ and its most important terms are $^{10-12}$ )

$$
V_{\mathrm{BF}}=\sum_{j} A_{j} \hat{n}_{\mathrm{d}} \hat{n}_{j}+\sum_{j j^{\prime}} \Gamma_{j j^{\prime}}\left(Q_{\mathrm{B}}^{(2)} \cdot\left(a_{j}^{\dagger} \tilde{a}_{j^{\prime}}\right)^{(2)}\right)+\sum_{j j^{\prime} j^{\prime \prime}} \Lambda_{j j^{\prime}}^{j^{\prime \prime \prime}}:\left(\left(d^{\dagger} \tilde{a}_{j}\right)^{\left(j^{\prime \prime}\right)} \times\left(d a_{j^{\prime}}^{\dagger}\right)^{\left(j^{\prime \prime}\right)}\right)_{0}^{(0)}
$$


with

$$
Q_{\mathrm{B}}^{(2)}=\left(s^{\dagger} \tilde{d}+d^{\dagger} s\right)^{(2)}+\chi\left(d^{\dagger} \tilde{d}\right)^{(2)}
$$

consisting of a monopole, quadrupole and an exchange force respectively. In order to restrict the model space which is outlined before (containing 155 parameters), semi-microscopic arguments are used to relate these parameters with the underlying quasi-particle structure of the odd particle. One obtains the following expressions ${ }^{8}$ ):

$$
\begin{gathered}
\Gamma_{j j^{\prime}}=\Gamma_{0}\left(u_{j} u_{j^{\prime}}-v_{j} v_{j^{\prime}}\right) Q_{j j^{\prime}} \\
\Lambda_{j j^{\prime}}^{j^{\prime \prime}}=-2 \sqrt{5} \Lambda_{0} \beta_{j j^{\prime}} \beta_{j^{\prime \prime} j^{\prime}} / \sqrt{2 j^{\prime \prime}+1}\left(E_{j}+E_{j^{\prime \prime}}-\hbar \omega\right) \\
\beta_{j j^{\prime}}=\left(u_{j} v_{j^{\prime}}+v_{j} u_{j^{\prime}}\right) Q_{j j^{\prime}}
\end{gathered}
$$

with $Q_{j j^{\prime}}$ defined as $\left\langle j\left\|Y_{2}\right\| j^{\prime \prime}\right\rangle$, and $v_{j}^{2}$ are the occupation probabilities $\left(u_{j}^{2}=1-v_{j}^{2}\right)$. The energy denominator $E_{j}+E_{j^{\prime \prime}}-\hbar \omega$ takes into account the non-degenerate single-particle orbitals in obtaining the energy of the D-state with respect to the Sstate. The hamiltonian (1) has been diagonalized with the use of the computer program ODDA [ ref. $\left.\left.^{13}\right)\right]$.

\subsection{DETERMINATION OF THE PARAMETERS}

In the present study, we concentrate on the odd-mass $\mathrm{Rh}$ isotopes, which are considered as resulting from coupling a proton hole to the even-even $\mathrm{Pd}$ nuclei. The latter nuclei have already been studied in much detail by Van Isacker et al. ${ }^{14}$ ) in the IBM-2. Since in the IBFM-1 no distinction is made between neutron and proton bosons, the IBM-1 parameters were obtained by projecting the IBM-2 hamiltonian onto the IBM-1 space and equating the matrix elements of the hamiltonian between states that are fully symmetric in the neutron-proton degree of freedom ${ }^{8}$ ). The remaining parameters appearing in the boson-fermion interaction $V_{\mathrm{BF}}\left(A, \Gamma_{0}, \Lambda_{0}\right)$ were determined starting from the values obtained in studying the odd-mass $\mathrm{Tc}$ isotopes ${ }^{1}$ ). They were subsequently adjusted in order to obtain a good description of both positive-parity and negative-parity states at the same time. Thereby one finally obtains the values $A=-0.25 \mathrm{MeV}, \Gamma_{0}=0.3 \mathrm{MeV}$ and $\Lambda_{0}=2.125 \mathrm{MeV}^{2}$. The value of $h \omega$ used is $1.5 \mathrm{MeV}$, which is consistent with the excitation energy for the $J^{\pi}=2_{1}^{+}$states in the even-even $\mathrm{Sn}$ isotopes. These parameters were kept constant for all $\mathrm{Rh}$ isotopes. In the study of the $\mathrm{Tc}$ isotopes the value of $\chi$ was determined by

$$
\chi=\frac{1}{2}\left(\chi_{\pi}+\chi_{\nu}\right)
$$


with $\chi_{\pi}$ and $\chi_{\nu}$ taken from the even-even Pd-core description. Using this relation for $\chi$ we obtained a poor agreement between experimental and theoretical spectroscopic factors for one-nucleon transfer. Instead of using eq. (5), we used the following expression:

$$
\chi=\chi_{v}
$$

which is the expression one obtains when assuming that the quadrupole BF interaction results from a proton-neutron interaction. In this way a much better description of the experimental spectroscopic factors is obtained. The values for $\chi$, $\Gamma_{0}$ and $A$ used in the present paper differ from the corresponding values used in the description of the odd-mass Tc nuclei ${ }^{1}$ ). One has to recall, however, that in the vibrational limit the monopole force compresses the energy spectrum without changing the wave functions. Furthermore it is the product $\chi \Gamma_{0}$ which determines the strength of the quadrupole force for the term $\left(d^{\dagger} \mathcal{d}\right)^{(2)}$, and this product is nearly the same as in ref. $\left.{ }^{1}\right)$. So, the only major difference occurs in the $\left(s^{\dagger} d\right)^{(2)}+\left(d^{\dagger} s\right)^{(2)}$ part of the quadrupole force which seems to be of particular importance in determining the spectroscopic factors (see eq. (9)). The occupation probabilities $v_{j}^{2}$ in eq. (4) are not free parameters. They were determined from BCS calculations starting from the single-particle energies $\varepsilon_{j}$. The proton single-particle energies for the $1 \mathrm{~g}_{\frac{2}{2}}, 2 \mathrm{p}_{\frac{1}{2}}, 2 \mathrm{p}_{\frac{2}{2}}$ and $1 \mathrm{f}_{\frac{s_{2}}{2}}$ orbitals were obtained, starting from the Nilsson model applied to fp-shell nuclei for the $A \simeq 100$ mass region ${ }^{15}$ ), by slightly adjusting in order to obtain a best fit to the experimental spectra. In general, the agreement between theory and experiment could be improved when also the $2 \mathrm{~d}_{\frac{3}{2}}$ orbit was included. The relative position of this orbital with respect to the $1 \mathrm{~g}_{\frac{2}{2}}$ orbit was taken from the proton separation energies ${ }^{16}$ ) resulting in a value of $4.5 \mathrm{MeV}$. The gap parameter $\Delta$ was taken as $1.5 \mathrm{MeV}$. In table 1 we list the single-particle energies $\varepsilon_{j}$, the quasiparticle energies $E_{j}$, the quasiparticle occupation probabilities $v_{j}^{2}$ and the parameters of $V_{\mathrm{BF}}$. These values were kept constant when calculating the energy spectra.

TABLE 1

The parameters of the hamiltonian (eq. (1)) as used in the present calculation

\begin{tabular}{cccc}
\hline$n l j$ & $\varepsilon_{j}$ & $E_{j}$ & $v_{j}^{2}$ \\
\hline $2 \mathrm{p}_{1 / 2}$ & -1.15 & 2.380 & 0.8882 \\
$2 \mathrm{p}_{3 / 2}$ & -2.00 & 3.086 & 0.9370 \\
$1 \mathrm{f}_{5 / 2}$ & -2.15 & 3.218 & 0.9424 \\
$1 \mathrm{~g}_{9 / 2}$ & 0.50 & 1.513 & 0.5653 \\
$2 \mathrm{~d}_{5 / 2}$ & 5.00 & 4.557 & 0.0279 \\
\hline
\end{tabular}

$$
A=-0.25 \mathrm{MeV} ; \Gamma_{0}=0.3 \mathrm{MeV} ; A_{0}=2.125 \mathrm{MeV}^{2} ; \hbar \omega=1.5 \mathrm{MeV} .
$$




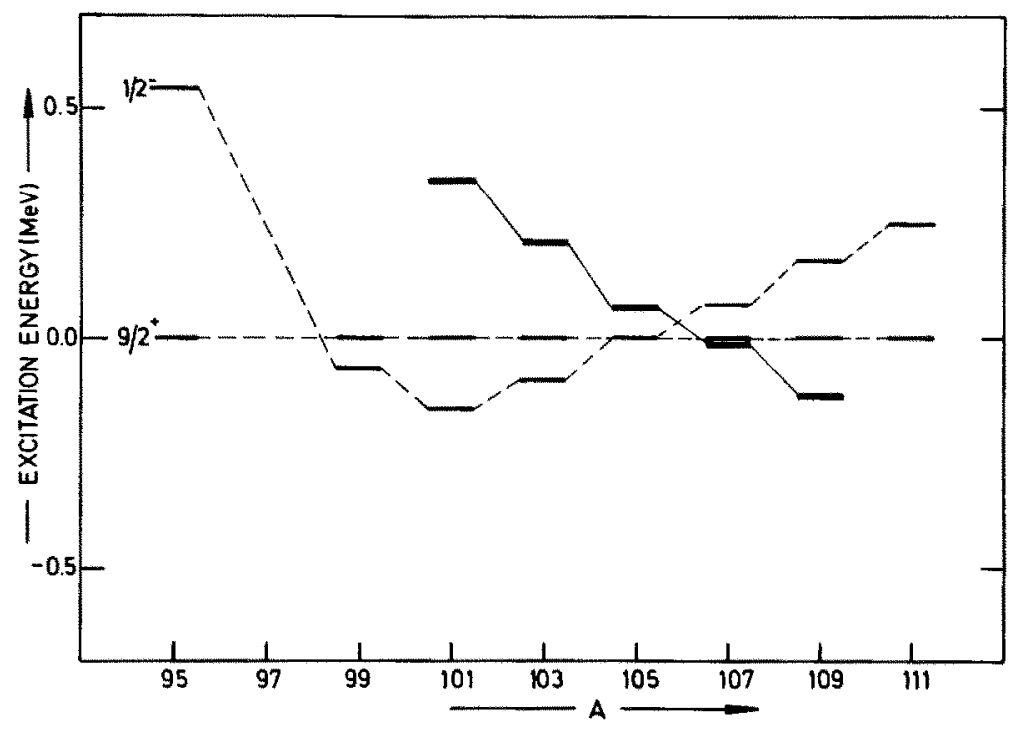

Fig. 1. The experimental relative position of the $J^{\pi}=\frac{1-}{2}$ level with respect to the $J^{\pi}=\frac{9+}{2}$ level throughout the odd-mass $\mathrm{Rh}$ isotopes ${ }^{17}$ ). The theoretical results for the calculated $\mathrm{Rh}$ isotopes are shown and connected with a solid line.

\section{Energy spectra}

Although both positive- and negative-parity levels were calculated with the same hamiltonian, it was not possible to reproduce the experimental energy difference between the lowest $J^{\pi}=\frac{9}{2}^{+}$and $J^{\pi}=\frac{1}{2}^{-}$states. In fig. 1 the position of the $J^{\pi}=\frac{1}{2}^{-}$state with respect to the $J^{\pi}=\frac{9}{2}^{+}$state is shown ${ }^{17}$ ). In the experimental data, the same behaviour as in the $\mathrm{Ag}$ isotopes ${ }^{18}$ ) is observed. However, the theoretical excitation energy for the $J^{n}=\frac{1}{2}^{-}$level decreases as a function of $N$, contrary to the experimental situation. Moreover the calculated crossing of the $J^{\pi}=\frac{1}{2}^{-}$and $J^{\pi}=\frac{9}{2}^{+}$levels is displaced towards neutron-rich nuclei with respect to the experimental $J^{\pi}=\frac{1}{2}^{-}, J^{\pi}=\frac{9}{2}+$ crossing.

The experimental positive-parity states indicate a transition from a particlevibration coupled spectrum in ${ }^{101} \mathrm{Rh}$ towards a rather complex spectrum, with increasing neutron number. Already in ${ }^{101} \mathrm{Rh}$ the $\left|2_{1}^{+} \times 1 \mathrm{~g}_{\frac{3}{2}} ; J^{\pi}\right\rangle$ quintuplet obtained in weak-coupling near the energy of the $I^{\pi}=2_{1}^{+}$state in the even-even Pd nuclei is highly perturbed. A very low-lying $J^{\pi}=\frac{7}{2}^{+}$results near the $J^{\pi}=\frac{9}{2}^{+}$ground state; a state that even becomes the ground state for the heavier odd-mass $\mathrm{Rh}$ nuclei. In the present calculations this transition is taken automatically in account by the changing structure of the even-even core with increasing neutron number. The $\mathbf{P d}$ isotopes represent a possible $\mathrm{U}(5) \rightarrow \mathrm{O}(6)$ transition ${ }^{14}$ ). In the IBFM-1 this 
becomes a transition from particle-vibration-coupled spectra (described by $\left.\mathrm{U}(5) \times 1 \mathrm{~g}_{\frac{9}{2}}\right)$ towards $\gamma$-unstable rotor plus particle spectra $\left(\mathrm{O}(6) \times 1 \mathrm{~g}_{\frac{9}{2}}\right)$.

In order to obtain a better agreement between calculated spectra and the experimental results we included the $2 \mathrm{~d}_{\frac{3}{2}}$ orbital. The effect of enlarging the model space is mainly an increase in excitation energy for the $J^{\pi}=\frac{11}{2}+$ and $\frac{13}{2}+$ levels and the high-spin states built on them. The disadvantage of including the $2 \mathrm{~d}_{\text {s. }}$ orbital is that the first $J^{n}=\frac{5}{2}^{+}$state results too low in excitation energy.

With a constant boson-fermion interaction $V_{\mathrm{BF}}$ it is thus possible to describe (i) the $J=j-1$ ground-state anomaly, (ii) the high-spin states, and (iii) the changing level structure from particle-vibration like towards more complex spectra, with increasing neutron number (fig. 2).

Preliminary results ${ }^{26}$ ) indicate that there may be evidence for low-lying $J^{\pi}=\frac{1}{2}^{+}$ and $\frac{3}{2}^{+}$intruder states in the $R h$ isotopes. Since the IBFM cannot reproduce intruder states, we have not included these levels in fig. 2 . A second state that could

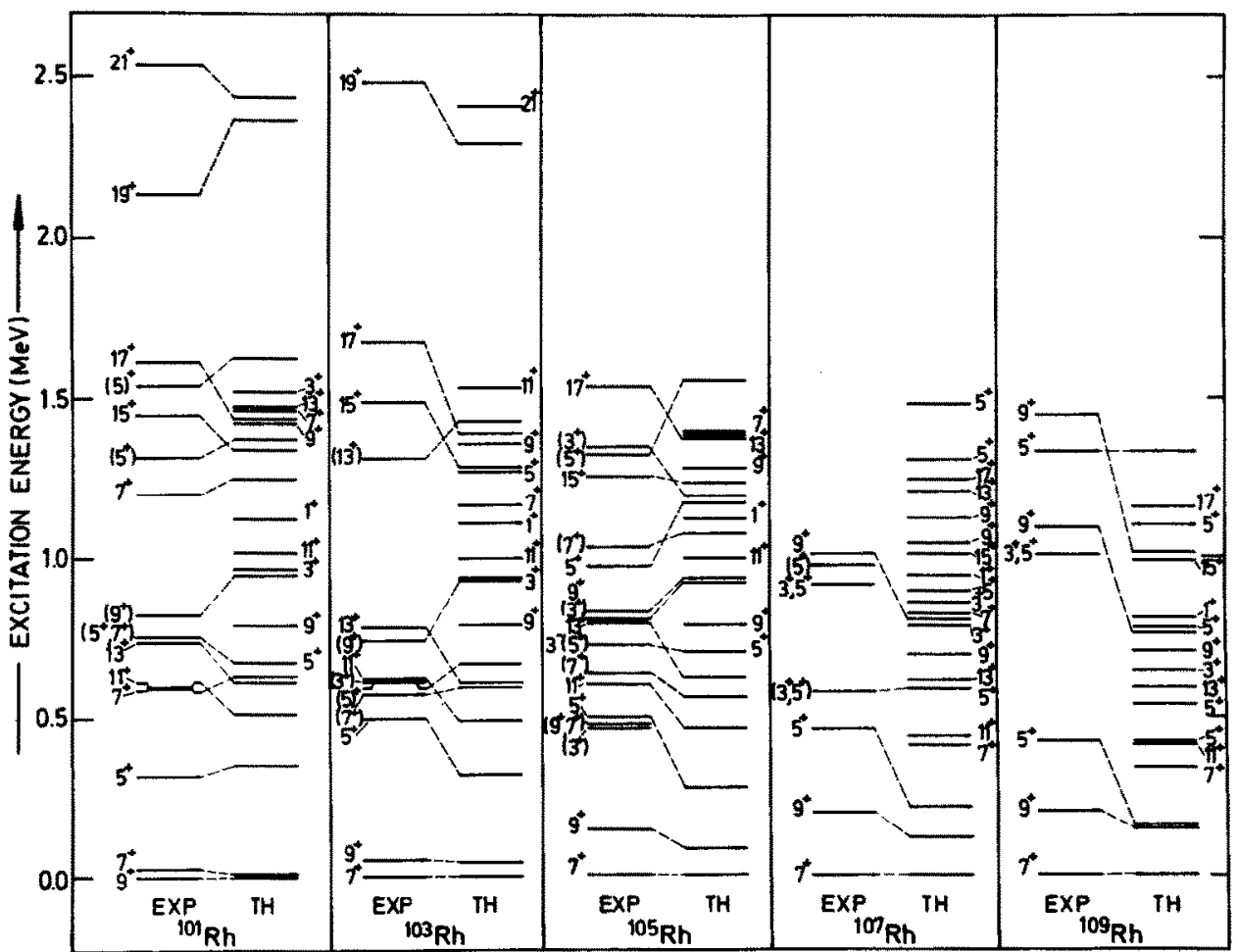

Fig. 2. The theoretical and experimental ${ }^{19-25}$ ) results for the low-lying positive-parity levels. The theoretical levels that cannot at present be connected with observed levels are also shown with their spin values. To simplify the figure only the lowest of these levels are shown. All indicated spin values are 
not be explained is a $J^{\pi}=\left(\frac{3}{2}{ }^{+}\right)$state in ${ }^{103} \mathrm{Rh}$ at $E_{\mathrm{x}}=0.612 \mathrm{MeV}$ for which the excitation energy decreases with increasing neutron number. Although the experimental assignment is not yet unique, the observation of another low-spin state in the neutron-deficient $\mathrm{Rh}$ isotopes, at this energy is not predicted by the IBFM-1 calculation. Further experimental data could help to give an interpretation of such a $J^{\pi}=\left(\frac{3}{2}^{+}\right)$state.

In fig. 3 the results for the lowest negative-parity states are shown. The main characteristic of the low-lying spectrum is the observation of three doublets: two with spin $J^{\pi}=\frac{3}{2}^{-}$and $\frac{5}{2}^{-}$and one with spin $J^{\pi}=\frac{7}{2}^{-}$and $\frac{9}{2}^{-}$. This doublet structure suggests a possible pseudospin symmetry ${ }^{27}$ ). The negative-parity states have been described ${ }^{28}$ ) recently in the framework of $\mathrm{U}(6 / 12)\left[\right.$ ref. $\left.\left.^{29}\right)\right]$, which incorporates this pseudospin symmetry. The lowest $J^{\pi}=\frac{3}{2}^{-}, \frac{5}{2}-$ doublet and the $J^{\pi}=\frac{7}{2}^{-}, \frac{9}{2}^{-}$doublet can be interpreted as the $2 \mathrm{p}_{\frac{1}{2}}$ particle coupled with the $I^{\pi}=2_{1}^{+}$and the $I^{\pi}=4_{1}^{+}$states of the even-even core. The second $J^{\pi}=\frac{3}{2}^{-}, \frac{5}{2}^{-}$

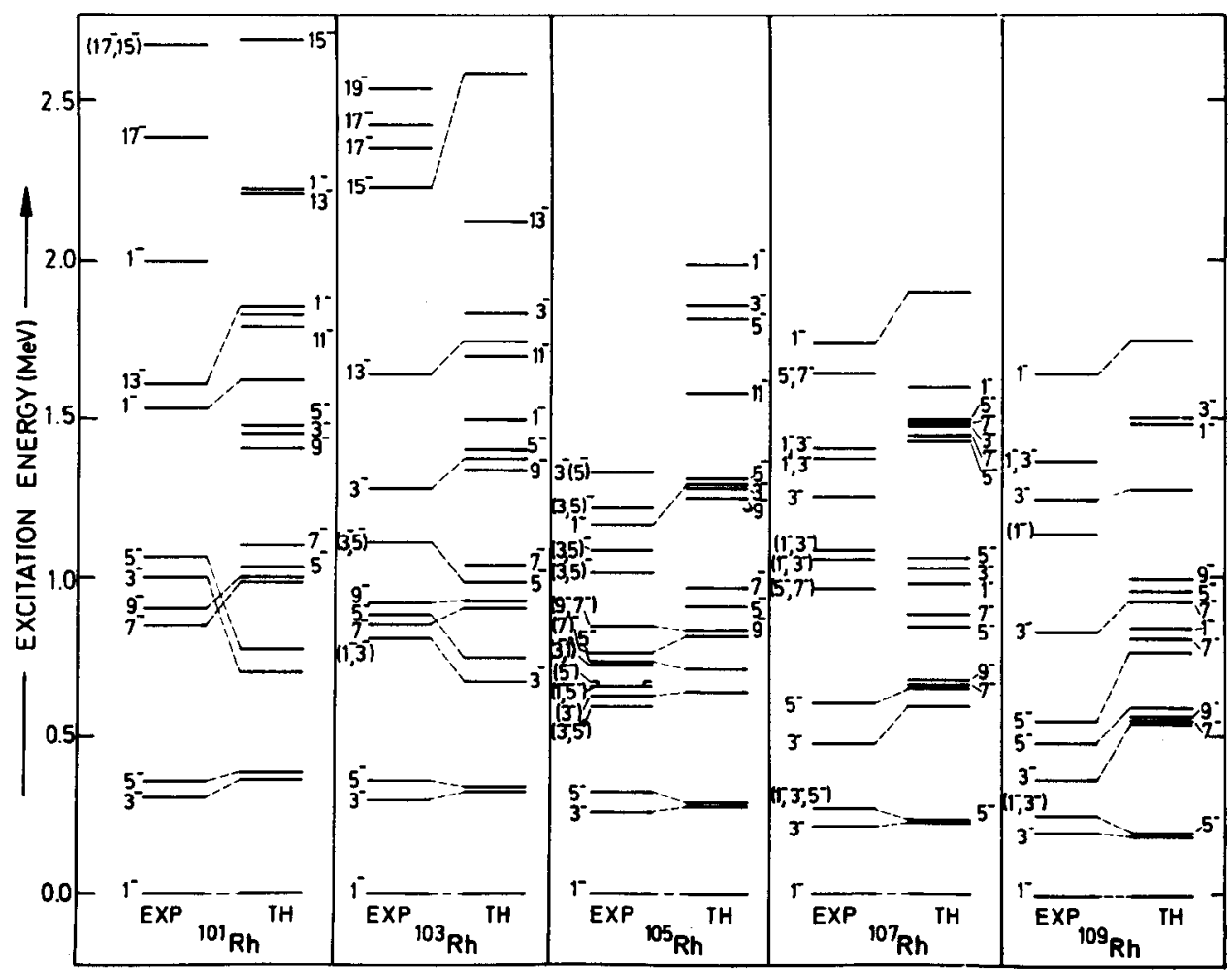

Fig. 3. The theoretical and experimental ${ }^{19-25}$ ) results for the low-lying negative-parity levels. The theoretical levels that cannot at present be connected with observed levels are also shown with their spin values. To simplify the figure only the lowest of these levels are shown. All indicated spin values are double-valued. 
doublet arises from coupling the $2 \mathrm{p}_{\frac{3}{2}}$ and $1 \mathrm{f}_{\frac{g_{2}}{2}}$ particles to the $I^{\pi}=0^{+}$ground state of the even-even core. The excitation energies of these doublets decreases with increasing neutron number. Theoretically we find also a decrease for the $J^{\pi}=\frac{7}{2}^{-}$ and $\frac{9}{2}-$ levels in the neutron-rich isotopes where no experimental data are available. In the theoretical spectrum a third $J^{n}=\frac{3}{2}-\frac{5}{2}-$ doublet, formed by coupling the $2 \mathrm{p}_{\frac{1}{2}}$ particle to the $I^{\pi}=2_{2}^{+}$state, occurs at a high excitation energy in ${ }^{101} \mathrm{Rh}$. With increasing neutron number the excitation energy of this doublet decreases from $E_{\mathrm{x}}=1.45 \mathrm{MeV}$ to $E_{\mathrm{x}}=0.9 \mathrm{MeV}$. Experimental data for this doublet, however, are not complete. In ${ }^{101} \mathrm{Rh}$ and ${ }^{103} \mathrm{Rh}$, it is not seen at all below $1 \mathrm{MeV}$ and in ${ }^{105} \mathrm{Rh}$ several levels occur at low energy, but with no clear spin assignments. The position of this third $J^{n}=\frac{3}{2}^{-}, \frac{5}{2}^{-}$doublet, however, is of importance because the major difference between the present IBFM-1 calculation and the $\mathrm{U}(6 / 12)$ calculation $\left.{ }^{28}\right)$, is that $\mathrm{U}(6 / 12)$ predicts the second and the third $J^{\pi}=\frac{3}{2}^{-}, \frac{5}{2}^{-}$doublet to be almost degenerate.

The high-spin states in ${ }^{101} \mathrm{Rh}$ and ${ }^{103} \mathrm{Rh}$ are calculated at a too high excitation energy. Using a single hamiltonian and thus the same moment of inertia for both positive- and negative-parity bands is possible at the origin of this discrepancy.

\section{Electromagnetic E2 transitions}

We have calculated E2 transition probabilities for the negative-parity levels of ${ }^{103} \mathrm{Rh}$. These electromagnetic transitions are the only known for the odd-mass $\mathrm{Rh}$ nuclei ${ }^{30}$ ).

The E2 transition operator in the IBFM is given by

$$
T_{\mathrm{B}}^{(2)}=e_{\mathrm{B}}\left(\left(s^{\dagger} \tilde{d}+d^{\dagger} \tilde{s}\right)^{(2)}+\chi_{\mathrm{t}}\left(d^{\dagger} \tilde{d}\right)^{(2)}\right)+e_{\mathrm{F}} \sum_{j j^{\prime}} Q_{j j^{\prime}}\left(a_{j}^{\dagger} \tilde{a}_{j}\right)^{(2)} .
$$

The first part of the operator is the standard boson E2 operator and the second part describes the contribution from the odd particle. On the basis of microscopic arguments ${ }^{11}$ ) one should expect an additional term in the E2 operator coming from exchange effects. In the present article this term has been omitted for simplicity. The parameter $\chi_{1}$ was taken equal to 0 which corresponds to the $O(6)$ value. Because microscopic calculations ${ }^{31}$ ) suggest that the boson and the fermion effective charges should be of the same magnitude, we have taken $e_{\mathrm{B}}=e_{\mathrm{F}}$ which leaves only one free parameter. The value of $e_{\mathrm{B}}$ was determined from the experimental $B\left(\mathrm{E} 2 ; \frac{3}{2}^{-} \rightarrow \frac{1}{2}^{-}\right)$value which is equal to $e_{\mathrm{b}}=0.104 e \cdot \mathrm{b}$. The $B\left(\mathrm{E} 2 ; J \rightarrow J^{\prime}\right)$ values in ${ }^{103} \mathrm{Rh}$ are listed in table 2 . The transitions for which two experimental numbers are given correspond to the two possible M1/E2 mixing ratios. 
TABLE 2

The experimental $\left.{ }^{23}\right)$ and theoretical $B\left(\mathrm{E} 2 ; J \rightarrow J^{\prime}\right)$ values for the negative-parity levels in ${ }^{103} \mathrm{Rh}$

\begin{tabular}{|c|c|c|}
\hline \multirow[t]{2}{*}{$J_{i}^{\pi} \rightarrow J_{f}^{\pi}$} & \multicolumn{2}{|c|}{$B\left(\mathrm{E} 2 ; J_{i}^{\pi} \rightarrow J_{f}^{\pi}\right)\left(e^{2} \cdot \mathrm{b}^{2}\right)$} \\
\hline & experiment & IBF M-1 \\
\hline$\frac{3}{2}-{ }_{1}^{-} \rightarrow \frac{1}{2}-$ & $0.109(8)$ & 0.110 \\
\hline$\frac{3}{2} 2-\frac{1}{2}-$ & unknown & 0.000 \\
\hline$\frac{5}{2}-\rightarrow \frac{1}{21}-$ & $0.118(7)$ & 0.110 \\
\hline$\frac{5}{2}-\frac{1}{2}-$ & $0.0044(3)$ & 0.000 \\
\hline$\frac{5}{22} \rightarrow \frac{3}{21}$ & $0.007(1)$ & 0.006 \\
\hline & $0.077(10)$ & \\
\hline$\frac{5-}{22} \rightarrow \frac{5-}{21}$ & $0.015(4)$ & 0.018 \\
\hline & $0.225(37)$ & \\
\hline$\frac{7}{21}-\rightarrow \frac{3}{2}-$ & $0.130(20)$ & 0.136 \\
\hline$\frac{9}{21}-\rightarrow \frac{5}{21}-$ & $0.181(15)$ & 0.151 \\
\hline
\end{tabular}

\section{Spectroscopic factors}

Both for positive- and negative-parity levels, single-particle transfer reactions were performed ${ }^{19,20}$ ) populating levels in ${ }^{105} \mathrm{Rh},{ }^{107} \mathrm{Rh}$ and ${ }^{109} \mathrm{Rh}$. These pick-up reactions provide experimental knowledge on the single-particle component of the wave function of a given state by means of the measured spectroscopic factor $C^{2} S$ :

$$
C^{2} S_{l j}\left(J_{\mathrm{i}}^{\pi} \rightarrow J_{\mathrm{f}}^{\pi}\right)=\mid\left\langle J_{\mathrm{f}}\left\|A_{i j}^{\dagger}\right\| J_{\mathrm{i}}\right\rangle^{2} .
$$

In order to calculate these spectroscopic factors one needs to specify the operator that connects the even-even nucleus with the adjacent odd-mass nucleus. Since in these reactions one goes from a Pd nucleus, with $N$ bosons (holes) to a $\mathrm{Rh}$ nucleus with the same number of bosons (holes) plus one fermion the operator takes, in first order, the following form ${ }^{8}$ ):

$$
A_{l j}^{\dagger}=\left(u_{j} a_{j}^{\dagger}-\sum_{j^{\prime}} \frac{v_{j}}{\sqrt{N_{\pi}}} \sqrt{\frac{10}{2 j+1}} \frac{N_{\pi}}{N} \beta_{j^{\prime} j}\left(K_{\beta}\right)^{-1} s^{\dagger}\left(d a_{j^{\prime}}^{\dagger}\right)^{(j)}\right) / K_{j},
$$

with $\beta_{j j^{\prime}}$ given by eq. (4c) and

$$
K_{\beta}^{2}=\sum_{j j^{\prime}} \beta_{j j^{\prime}}^{2},
$$

and $K_{j}$ is a normalization coefficient determined by the condition

$$
\sum_{\text {odd }}\left\langle\text { odd }\left|A_{l j}^{\dagger}\right| \text { even }\right\rangle^{2}=(2 j+1) u_{j}^{2}
$$


TABLE 3

The experimental and calculated spectroscopic factors for one-nucleon pick-up starting from the eveneven Pd nuclei leading towards the positive-parity levels in ${ }^{105} \mathrm{Rh},{ }^{107} \mathrm{Rh}$ and ${ }^{109} \mathrm{Rh}$, the experimental reaction used was $\operatorname{Pd}(\vec{t}, \alpha) \operatorname{Rh}\left[\right.$ ref. $\left.\left.^{24}\right)\right]$

\begin{tabular}{|c|c|c|c|c|c|}
\hline & \multirow[b]{2}{*}{$J^{\pi}$} & \multicolumn{2}{|c|}{ Experiment } & \multicolumn{2}{|c|}{ IBFM-1 } \\
\hline & & $E_{\mathrm{x}}$ & $C^{2} S$ & $E_{\mathrm{x}}$ & $C^{2} S$ \\
\hline \multirow[t]{4}{*}{${ }^{105} \mathrm{Rh}$} & $\frac{7}{2}^{+}$ & 0 & $(0.21)$ & 0 & 0.00 \\
\hline & $\frac{9}{2}+$ & 152 & 4.1 & 82 & 4.32 \\
\hline & $\frac{9}{2}+$ & 830 & 1.3 & 931 & 0.79 \\
\hline & $\frac{7}{2}^{+}$ & 1024 & $(0.45)$ & 1075 & 0.00 \\
\hline \multirow[t]{3}{*}{${ }^{107} \mathrm{Rh}$} & $\frac{7}{2}^{+}$ & 0 & $(0.26)$ & 0 & 0.00 \\
\hline & $\frac{9}{2}+$ & 194 & 4.0 & 120 & 3.97 \\
\hline & $\frac{9}{2}+$ & 1000 & 2.3 & 809 & 0.76 \\
\hline \multirow[t]{6}{*}{${ }^{109} \mathrm{Rh}$} & $\frac{7}{2}^{+}$ & 0 & 0.05 & 0 & 0.00 \\
\hline & $\frac{9}{2}+$ & 206 & 3.7 & 148 & 3.30 \\
\hline & $\frac{5}{2}+$ & 424 & $(0.21)$ & 155 & 0.012 \\
\hline & $\frac{\bar{y}}{2}+$ & 1040 & 1.9 & 761 & 0.92 \\
\hline & $\frac{5}{2}+$ & 1330 & $(0.11)$ & 1336 & 0.00 \\
\hline & $\frac{9}{2}+$ & 1449 & $(0.40)$ & 1013 & 0.42 \\
\hline
\end{tabular}

where the summation runs over all levels of a given angular momentum in the odd-mass nucleus.

The calculated and experimental spectroscopic factors for pick-up to the positive-parity states are given in table 3. The agreement is good with the exception of the $J^{\pi}=\frac{9}{2}^{+}$state observed at $E_{\mathrm{x}}=0.83,1.00,1.04 \mathrm{MeV}$ in ${ }^{105} \mathrm{Rh}$, ${ }^{107} \mathrm{Rh}$ and ${ }^{109} \mathrm{Rh}$, respectively. We underestimate the spectroscopic factor of this level.

The spectroscopic factors for pick-up towards the low-lying $J^{\pi}=\frac{1}{2}^{-}, \frac{3}{2}^{-}, \frac{5}{2}^{-}$ negative-parity levels are shown in fig. 4. Taking into account an uncertainty of $25 \%$ on the experimental data, there is a good qualitative agreement. If we now consider the ratio

$$
r=\frac{C^{2} S_{\mathrm{f}_{5 / 2}}\left(0^{+}\right.}{C^{2} S_{\mathrm{p}_{3 / 2}}\left(0^{+}\right.} \frac{\left.\frac{5}{2}^{-}\right)}{\left.\rightarrow \frac{3}{2}^{-}\right)}
$$

for the two lowest $J^{\pi}=\frac{3}{2}^{-}, \frac{5}{2}^{-}$doublets (see table 4), the experimental values differ substantially whereas the IBFM-1 calculation gives an almost constant value for this ratio. Also, the calculation for pick-up spectroscopic factors in ref. ${ }^{28}$ ), using the $\mathrm{U}(6 / 12)$ scheme, predicts the same ratio for the two doublets, namely $r=1.5$. To understand the discrepancy between experiment and calculated ratios, we have included the $1 f_{\frac{7}{2}}$ orbital in the IBFM-1 calculation and assumed it to be completely 
filled $\left(v_{j}^{2}=1\right)$ at a quasiparticle energy of $E_{\mathrm{x}}=6.5 \mathrm{MeV}$. As shown in fig. 4, we then obtain a better agreement for the spectroscopic factors, except for an underestimation of $C^{2} S_{\mathrm{f}_{3 / 2}}\left(0^{+} \rightarrow \frac{5}{2}-\right)$. Incorporating this $1 \mathrm{f}_{\frac{7}{2}}$ orbital in the IBFM-1 model space also gives a splitting of the two $J^{\pi}=\frac{5}{2}^{-}$to $\frac{3}{2}^{-}$ratios $r$, defined in eq. (12) (see table 4), with, however, too low a value for the second doublet.

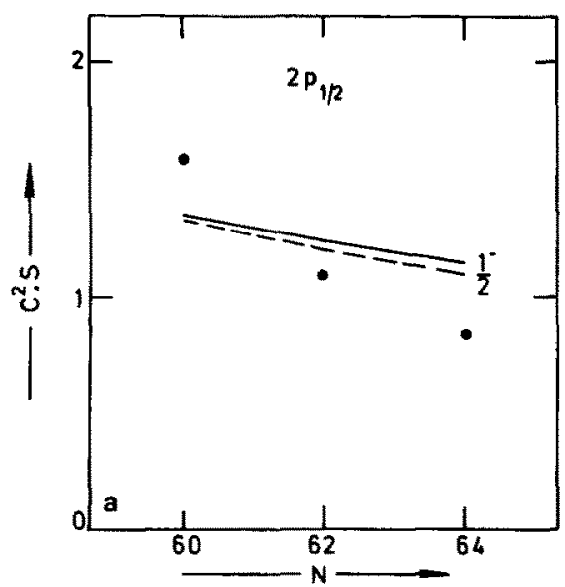

Fig. 4a. The experimental one-nucleon pick-up spectroscopic factors for the first $J^{n}=\frac{1}{2}^{-}$level (filled circles). The calculated values are connected with a solid line. The dashed line shows the results for the calculation when incorporating the $1 \mathrm{f}_{7 / 2}$ orbit. The reactions used were: $\operatorname{Pd}(\overrightarrow{\mathrm{t}}, \alpha) \mathrm{Rh}$ for $\left.{ }^{105} \mathrm{Rh}\left[\mathrm{ref} .{ }^{24}\right)\right]$ and $\mathrm{Pd}\left(\mathrm{d},{ }^{3} \mathrm{He}\right) \mathrm{Rh}$ for ${ }^{107} \mathrm{Rh}$ and ${ }^{109} \mathrm{Rh}$ [ref. $\left.\left.{ }^{25}\right)\right]$.

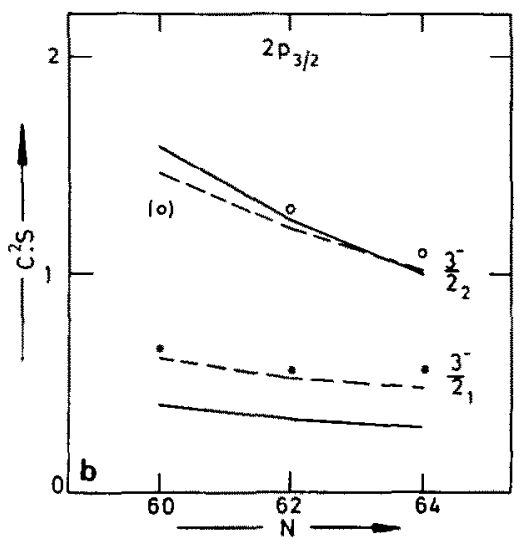

Fig. 4b. The experimental one-nucleon pick-up spectroscopic factors for the first $J^{n}=\frac{3}{2}^{-}$level (filled circles) and the second $J^{n}=\frac{3^{-}}{2}$ level (open circles). The calculated values are connected with solid lines. The dashed lines show the results for the calculation when incorporating the $1 \mathrm{f}_{7 / 2}$ orbit. The reactions used were: $\mathrm{Pd}(\overrightarrow{\mathrm{t}}, \alpha) \mathrm{Rh}$ for $\left.{ }^{105} \mathrm{Rh}\left[\mathrm{ref} .{ }^{24}\right)\right]$ and $\mathrm{Pd}\left(\mathrm{d},{ }^{3} \mathrm{He}\right) \mathrm{Rh}$ for ${ }^{107} \mathrm{Rh}$ and $\left.{ }^{109} \mathrm{Rh}\left[\mathrm{ref} .{ }^{25}\right)\right]$. 


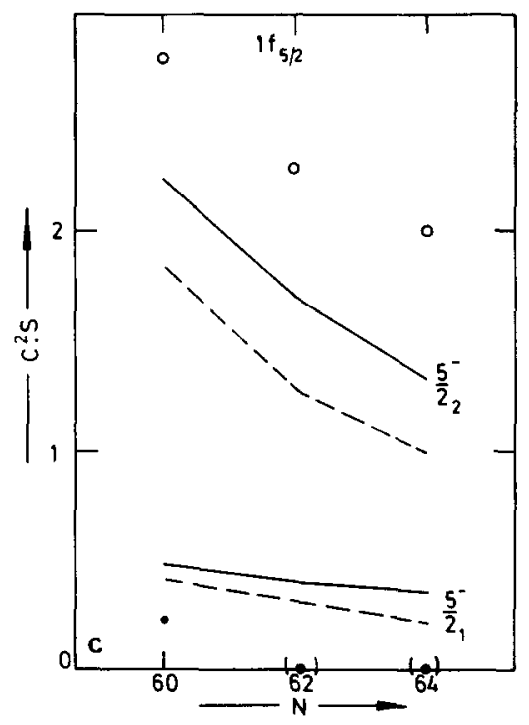

Fig. 4c. The experimental one-nucleon pick-up spectroscopic factors for the first $J^{\pi}=\frac{5}{2}-$ level (filled circles) and the second $J^{\pi}=\frac{5}{2}-$ level (open circles). The calculated values are connected with solid lines. The dashed lines show the results for the calculation when incorporating the $1 f_{7 / 2}$ orbit. The reactions used were: $\mathrm{Pd}(\overrightarrow{\mathrm{t}}, \alpha) \mathrm{Rh}$ for ${ }^{105} \mathrm{Kh}$ [ref. $\left.\left.{ }^{24}\right)\right]$ and $\mathrm{Pd}\left(\mathrm{d},{ }^{3} \mathrm{He}\right) \mathrm{Rh}$ for ${ }^{107} \mathrm{Rh}$ and $\left.{ }^{109} \mathrm{Rh}\left[\mathrm{ref} .{ }^{25}\right)\right]$.

TABLE 4

The ratio $r=C^{2} S_{\mathrm{f}_{5 / 2}}\left(0^{+} \rightarrow \frac{5}{2}^{-}\right) / C^{2} S_{\mathrm{p}_{3 / 2}}\left(0^{+} \rightarrow \frac{3}{2}^{-}\right)$of the two lowest $J^{\pi}=\frac{5}{2}^{-}, \frac{3}{2}^{-}$doublets in ${ }^{105} \mathrm{Rh}$, ${ }^{107} \mathrm{Rh}$ and ${ }^{109} \mathrm{Rh}$

\begin{tabular}{|c|c|c|c|c|}
\hline \multirow[t]{2}{*}{ Ratio } & \multirow[t]{2}{*}{$A$} & \multirow[t]{2}{*}{ Experiment } & \multicolumn{2}{|c|}{ IBFM-I } \\
\hline & & & without $1 f_{7 / 2}$ & with $1 f_{7 / 2}$ \\
\hline \multirow{3}{*}{$r_{1}=\frac{C^{2} S\left(\frac{5}{2}-1\right)}{C^{2} S\left(\frac{3}{2}-1\right)}$} & $\int 105$ & 0.35 & 1.21 & 0.68 \\
\hline & $\{107$ & $(0.15)$ & 1.24 & 0.67 \\
\hline & $(109$ & $(0.11)$ & 1.28 & 0.68 \\
\hline \multirow{3}{*}{$r_{2}=\frac{C^{2} S\left(\frac{5}{22}\right)}{C^{2} S\left(\frac{3}{22}\right)}$} & $\int 105$ & $(2.15)$ & 1.43 & 1.26 \\
\hline & $\{107$ & 1.76 & 1.36 & 1.06 \\
\hline & $(109$ & 1.82 & 1.30 & 0.90 \\
\hline
\end{tabular}

\section{Conclusion}

In the present study, we have shown that it is possible to describe the low-lying excited states in the odd-mass $\mathrm{Rh}$ isotopes within the framework of the IBFM-1. In 
contrast to previous calculations within the IBFM-1, the present calculations are based on a single hamiltonian for the description of both positive- and negativeparity states. Thereby the number of parameters becomes much restricted. The calculated $B(E 2)$ values and spectroscopic factors for one nucleon pick-up are in good agrecment with cxisting data.

The possibility of obtaining a description of both positive- and negative-parity levels, starting from a single hamiltonian is probably due to the following two factors. (i) The IBM-2 hamiltonian was obtained from detailed study of spectra and E2 electromagnetic properties of the even-even Pd nuclei. (ii) The singleparticle properties (quasiparticle energy $E_{j}$ and occupation probabilities $v_{j}^{2}$ ) have been determined from a BCS calculation for both the $1 \mathrm{~g}_{\frac{3}{2}}, 2 \mathrm{~d}_{\frac{3}{2}}$ and the $2 \mathrm{p}_{\frac{1}{2}}, 2 \mathrm{p}_{\frac{3}{2}}$ and $1 \mathrm{f}_{\frac{5}{2}}$ orbitals.

We studied also the influence of the inclusion of the $1 \mathrm{f}_{\frac{7}{2}}$ orbital. Although the energy spectra do almost not change, different features of the wave functions are sensitive to this admixture, improving the one nucleon pick-up spectroscopic factors with respect to only using the $2 \mathrm{p}_{\frac{1}{2}}, 2 \mathrm{p}_{\frac{3}{2}}, 1 \mathrm{f}_{\frac{5}{2}}$ orbitals for the negative-parity states.

We would like to thank J. Vervier and M. Loiselet for valuable discussion, M. Harakeh for a critical reading of an early draft of the manuscript and D. De Frenne and E. Jacobs for the use of experimental results on ${ }^{107} \mathrm{Rh}$ and ${ }^{109} \mathrm{Rh}$ prior to publication. Three of the authors (P.V.I, J.M, M.W.) want to thank the NFWO (Nationaal fonds voor Wetenschappelijk Onderzoek) and one of us (J.J.) the IWONL (Instituut ter aanmoediging van het Wetenschappelijk Onderzoek in de Nijverheid en Landbouw) for financial support. O.S. thanks the National Science Foundation which supported this work under grant no. PHY-83-12245.

\section{References}

1) P. De Gelder, D. De Frenne, K. Heyde and N. Kaffrell, Nucl. Phys. A401 (1983) 397

2) A. Arima and F. Iachello, Ann. of Phys. 99 (1976) 253

3) A. Arima and F. Iachello, Ann. of Phys. 111 (1978) 201

4) A. Arima and F. Iachello, Ann. of Phys. 123 (1979) 468

5) A. Arima, T. Otsuka, F. Iachello and I. Talmi, Phys. Lett. 66B (1977) 205

6) T. Otsuka, A. Arima, F. Iachello and I. Talmi, Phys. Lett. 76B (1978) 139

7) F. Iachello and O. Scholten, Phys. Rev. Lett. 43 (1979) 679

8) O. Scholten, Ph.D. thesis, University of Groningen, The Netherlands (1980), unpublished

9) M. A. Cunningham, Nucl. Phys. A385 (1982) 204, 221

10) O. Scholten and N. Blasi, Nucl. Phys. A380 (1982) 509

11) O. Scholten and A. E. L. Dieperink, in Interacting Bose-Fermi systems in nuclei, ed. F. Iachello (Plenum, New York, 1981) p. 329

12) I. Talmi, in Interacting Bose-Fermi systems in nuclei, ed. F. Iachello (Plenum, New York, 1981) p. 343

13) O. Scholten, program package ODDA, KVI internal report no. 255 (1980)

14) P. Van Isacker and G. Puddu, Nucl. Phys. A348 (1980) 125 
15) S. E. Larsson, G. Leander, I. Ragnarsson and N. G. Alenius, Nucl. Phys. A261 (1976) 77

16) A. H. Wapstra and G. Audi, Nucl. Phys. A432 (1985) 1

17) Table of isotopes, 7th ed., ed. M. Lederer and V. Shirley (Wiley, New York, 1978)

18) M. Huyse, K. Cornelis, G. Lhersonneau, J. Verplanckeet and W. B. Walters, Nucl. Phys. A352 (1981) 247

19) E. R. Flynn, F. Ajzenberg-Selove, R. E. Brown, J. A. Cizewski and J. W. Sunier, Phys. Rev. C27 (1983) 2587

20) P. De Gelder, E. Jacobs, H. Thierens, K. Heyde, N. Kaffrell, N. Blasi, M. N. Harakeh and W. A. Sterrenburg, unpublished

21) G. Kayrys, M. Irshad, S. Landsberger, R. Lecomte, P. Paradis and S. Monaro, Phys. Rev. C26 (1982) 138

22) A. Charvet, R. Duffait, R. Beraud, K. Deneffe, A. Emsallem, M. Meyer, J. Treherne and A. Gizon, Z. Phys. A315 (1984) 163

23) B. Harmatz, Nucl. Data Sheets 28 (1979) 403

24) B. Harmatz, Nucl. Data Sheets 28 (1979) 343

25) G. Kayrys, S. Landsberger and S. Monaro, Phys. Rev. C28 (1983) 2335

26) E. Jacobs, private communication

27) O. Scholten, Phys. Lett. 108B (1982) 155

28) P. Van Isacker, J. Jolie, K. Heyde, M. Waroquier, J. Moreau and O. Scholten, Phys. Lett. 149B (1984) 26

29) A. B. Balantekin, I. Bars, R. Bijker and F. Iachello, Phys. Rev. C27 (1983) 1761

30) J. Vervier and R. Janssens, Phys. Lett. 108B (1982) 1

31) O. Scholten, Rev. Nucl. Part. Phys., to be published 